\title{
Hemodinamia y Anestesia
}

\section{Hemodynamics \& Anesthesia}

\author{
Pablo José Fiorito ${ }^{1, *}$ \\ 1 Servicio de Anestesiología. Hospital J.M. Ramos Mejía. Buenos Aires, Argentina.
}

Fecha de recepción: 16 de marzo de 2021 / Fecha de aceptación: 23 de agosto de 2021

\begin{abstract}
Introduction: Few medical specialties devote themselves to the constant monitoring and manipulation of hemodynamic variables such as Anesthesiology. Alongside the clinical context, monitoring by the anesthesiologist grants him information according to which he will tolerate the observed changes or take therapeutic actions in order to maintain homeostasis. The goal of this article is to arbitrarily review concepts of cardiovascular physiology, in order to achieve a correlation between them and relevant topics to the practice of Anesthesiology. Discussion: Pioneers in the field of Hemodynamics, Daniel Bernoulli, Jean Leonard Marie Poiseuille and Otto Frank established a great deal of our current knowledge of the discipline. Some physiologically based and poorly understood clinical dilemma include: - Autoregulation flow curves present in some organs, with their rigid and static concept ingrained by classical teachings, when there is evidence of the opposite in the patient under anesthesia; - Hemodynamics of the elderly, which requires a more complex analysis than that allowed by classical models; - The always problematic acute kidney injury (AKI), which prevention remains elusive. Conclusion: Knowledge of cardiovascular physiology will never stop being relevant. We must bear in mind its strengths, as well as its limitations. No matter his subspecialty of choice, the anesthesiologist will be daily up to the task of manipulating the hemodynamics of patients under his care. A sound physiological base will help in achieving better perioperative outcomes.
\end{abstract}

Key words: Hemodynamics, anesthesia, autoregulation curves, acute kidney injury.

\section{RESUMEN}

Introducción: Pocas especialidades como la anestesiología se dedican al monitoreo y manipulación constante de las variables hemodinámicas. Junto al contexto clínico, el monitor que el anestesiólogo vigila le otorga la información en base a la cual tolerará los cambios observados o tomará sus decisiones terapéuticas buscando el mantenimiento de la homeostasis. La finalidad de este artículo es revisar arbitrariamente algunos conceptos de la fisiología cardiovascular, para luego intentar realizar un correlato entre estos y tópicos de relevancia para la práctica de la anestesiología. Discusión: Pioneros en el terreno de la hemodinamia, Daniel Bernoulli, Jean Leonard Marie Poiseuille y Otto Frank sentaron las bases de gran parte de nuestro conocimiento actual de la disciplina. Algunos dilemas clínicos de base fisiológica pobremente entendidos en su totalidad y con relevancia a la práctica son: - Las curvas de autorregulación características de algunos órganos, cuya descripción clásica conlleva a un concepto rígido y estático de las mismas, cuando hay evidencias de lo contrario en el paciente anestesiado; - La hemodinamia de la población anciana, que requiere de un análisis más complejo que aquel permitido por modelos clásicos; - La siempre problemática injuria renal aguda, cuya prevención se mantiene elusiva. Conclusiones: Conocer la fisiología cardiovascular nunca dejará de ser relevante. Debemos tener en cuenta sus fortalezas, así como también sus limitantes. Sin importar la subespecialidad a la que se dedique, todo anestesiólogo se verá diariamente encargado de manejar la hemodinamia de los pacientes a su cargo. Una sólida base fisiológica ayudará a mejorar los desenlaces perioperatorios.

Palabras clave: Hemodinamia, anestesia, curvas de autorregulación, injuria renal aguda. 


\section{Introducción}

$\mathrm{P}$ ocas especialidades como la anestesiología se dedican al monitoreo y manipulación constante de las variables hemodinámicas. Junto al contexto clínico, el monitor que el anestesiólogo vigila le otorga la información en base a la cual tolerará los cambios observados o tomará sus decisiones terapéuticas buscando el mantenimiento de la homeostasis.

A pesar de la situación antifisiológica en conjunto a la que se encuentra expuesto el paciente bajo anestesia, es irónicamente un conocimiento sólido de la fisiología normal aquel que lo asistirá en su trabajo diario. La finalidad de este artículo es revisar arbitrariamente algunos conceptos de la fisiología cardiovascular, para luego intentar realizar un correlato entre estos y tópicos de relevancia para la práctica de la anestesiología.

\section{Principio de Bernoulli y ley de Hagen-Poiseuille}

Miembro de una ilustre familia de matemáticos, Daniel Bernoulli (1700-1782) investigó filosofía, medicina y lógica. Dentro de su extenso trabajo que incluyó campos tan diversos como la matemática, la fisiología, la astronomía y la oceanografía, su obra Hydrodinamica (1738) es probablemente la más recordada[1]. En ella desarrolla el principio que lleva su nombre.

La ecuación de Bernoulli, en una de sus formas más conocidas, es la siguiente:

$$
\rho \frac{1}{2} v^{2}+\rho g h+\rho g h=\text { constante }(1)
$$

Escrita de tal manera, la misma es aplicable al flujo continuo, incompresible y no viscoso de un fluido ideal. Al mantenerse constante la energía total del sistema por ausencia de pérdidas viscosas, el cambio en la magnitud de uno de los componentes (cinético, gravitacional y gravitacional potencial) es provocado a expensas de otro[2]. Su formulación suele ser utilizada en la descripción del efecto Venturi y como recordatorio que en realidad los fluidos no circulan de acuerdo a gradientes de presión, sino en concordancia a gradientes energéticos[3]. En la práctica clínica, la ecuación de Bernoulli modificada es utilizada en la ecocardiografía para la determinación de gradientes de presión a partir de velocidades de flujos.

Jean Léonard Marie Poiseuille (1797-1869), fisiólogo y físico francés, fue el inventor del manómetro en $U$ de mercurio e investigador experimental. Sus experimentos hicieron eco en múltiples disciplinas incluyendo la ingeniería, la física, la biología y la medicina. Luego de la realización de su tesis doctoral, Poiseuille se interesó en la hemodinamia en la microcirculación. Ante la imposibilidad de estudiar el tópico en modelos in vivo, realizó meticulosas mediciones en capilares de vidrio. Sus observaciones, dadas a conocer inicialmente en 1840, le valieron su ecuación homónima. Debido al trabajo independiente y contemporáneo publicado un año antes del ingeniero alemán Gotthilf Heinrich Ludwig Hagen (1797-1884), la ley es también conocida como la ecuación de Hagen-Poiseuille[4].

Por su parte, la ecuación de Poiseuille establece:

$\Delta \mathrm{P}=\mathrm{Q} R(2)$
Esta ecuación es a veces estimada de mayor relevancia que la de Bernoulli, enfatizando la imposibilidad de aplicar esta última en la totalidad de la circulación humana con la sangre -un fluido real y no ideal, propenso a pérdidas energéticas viscosas de mayor o menor cuantía de acuerdo con la sección del árbol vascular- fluyendo por la misma.

Es frecuentemente omitido el entorno experimental a partir del cual la ley fue derivada: un sistema de tubos rígidos de vidrio[4]. En consecuencia, suelen pasarse por alto las condiciones necesarias para su aplicación, no todas satisfechas por la circulación humana[3]. Dichas condiciones son:

- El fluido debe ser incompresible.

- El tubo debe ser derecho, rígido, cilíndrico, no ramificado y de radio constante.

- La velocidad de la capa de fluido en contacto con la pared debe ser 0 .

- Flujo laminar.

- Flujo no pulsátil.

- Viscosidad del fluido constante.

Un rápido repaso de estas condiciones nos revela que al pensar a la circulación en estos términos se pierden de vista características propias de la misma, como lo son su pulsatilidad o la presencia de segmentos vasculares complacientes y colapsables, entre otras que deben ser necesariamente omitidas.

\section{Presión, flujo, resistencia e impedancia}

Tal vez como un efecto colateral de la aplicación de la ecuación de Poiseuille, la presión arterial toma ventaja en el inconsciente colectivo como variable hemodinámica por encima del resto. De una manera parecida a la que la invención del esfingomanómetro desplazó al análisis de la onda de pulso a pesar de otorgar informaciones complementarias, su uso rutinario como parámetro vital de fácil accesibilidad probablemente la haya colocado en este lugar. Ya sea por limitaciones tecnológicas o por la búsqueda de la práctica menos invasiva posible sobre el paciente, la medición de flujos y su análisis han quedado en segundo plano.

Aún más, el flujo es comprendido como una variable dependiente de la presión pese a que, como dicho anteriormente, son los gradientes energéticos aquellos que lo determinan. Sin embargo, no debe subestimarse el efecto mecánico de la presión en determinados segmentos del sistema cardiovascular y en este caso sí se genera una dependencia clara del flujo en estos sectores. Podemos enunciar, a manera de ejemplo:

1) Los fenómenos de apertura y cierre de las válvulas cardíacas.

2) El cambio de diámetro de estructuras vasculares. El aumento de presión transmural redunda en una disminución de la resistencia vascular en ausencia de autorregulación. Llevado al extremo contrario, no habrá flujo posible en ausencia de una presión mínima necesaria para mantener patente la luz del vaso, la presión crítica de cierre. El interjuego entre la presión intraluminal y circundante es de relevancia fisiológica en la circulación miocárdica, cerebral y pulmonar. En la patología, encontramos los ejemplos del taponamiento cardíaco o la compresión vascular en masas mediastinales anteriores.

3) La filtración a nivel glomerular renal y en los capilares sistémicos, regida por la ecuación de Starling modificada. 
Sin presión no hay flujo, pero sin flujo tampoco hay presión. El fluido es el responsable de la fuerza realizada sobre la pared vascular. Es complejo intentar asignar independencia y dependencia de variables en el sistema cardiovascular. La igualdad matemática pouiseuilliana nos hace pensar en estos términos, cuando lo que evidencia es una relación entre tres variables. En cuanto a la resistencia, las confusiones son menores. Matemáticamente podemos obtener su valor a partir de la relación entre presión y flujo, pero también sabemos que los determinantes de la resistencia son:

$R=8 \mid \eta / \pi r^{4}(3)$

En su definición física, no debe olvidarse a la presión como una fuerza sobre un área:

$P=F / A(4)$

Mientras que el caudal depende de:

$Q=\vee A(5)$

Al tener en mente las ecuaciones (3), (4) y (5), la ecuación de Poiseuille no es una declaración de dependencias, sino de relaciones.

El concepto de impedancia otorga una caracterización del sistema arterial más compleja y precisa que el de resistencia. La clásica resistencia está incluida dentro de la misma, pero también están presentes la distensibilidad arterial, la inercia de la sangre y reflexiones de la onda de pulso[5]. En otro salto de simplificación, se asume al corazón como una bomba generadora de presión continua y se trazan analogías con un sistema de corriente continua. Más correcta es su comparación con uno de corriente alterna y la presencia de ondas complejas alternantes de flujo y presión, vinculadas entre sí por la impedancia del sistema. A un nivel menos abstracto podemos pensar en la interacción (acople) entre ventrículo y vasculatura, cuya resultante son los valores de flujo y presión generados. El enfoque es atractivo en especial para comprender los efectos del envejecimiento sobre el sistema cardiovascular. Conceptualmente, también ayuda a pensar en segmentos vasculares distensibles, no rígidos, y para tener en cuenta fenómenos inerciales. Su análisis se circunscribe a la porción arterial del sistema.

Pensar al corazón como una bomba no pulsátil generadora de presión continua y eyectando hacia tubos rígidos puede ser de utilidad como introducción inicial a la fisiología cardiovascular y deseable en aras de la simplicidad, pero es importante tener en cuenta que quizás haya situaciones que escapen a las respuestas que pueda otorgar un modelo alejado de la realidad.

\section{La ecuación de Bernoulli modificada}

En realidad, tanto la ecuación de Bernoulli como la de Poiseuille tienen sus limitaciones. Como ventaja, la primera tiene en cuenta el componente gravitacional y el acelerativodesacelerativo. Como desventaja, ignora las pérdidas viscosas. Lo contrario ocurre con la segunda.

Badeer propone que ambas son incompletas, y en su lugar sugiere la utilización de una ecuación combinada para lograr superar las limitantes de ambas, designada la ecuación de
Bernoulli-Poiseuille[2]. La adición de un término que tiene en cuenta las pérdidas viscosas permite recuperar el concepto de la expresión de la energía del fluido en distintos componentes, en especial el cinético que suele ser olvidado, y la posibilidad de interconversión entre ellos. El componente cinético toma relevancia en estados hiperdinámicos de la circulación.

Aún más interesante es la posibilidad de la derivación y aplicación de la ecuación de Bernoulli en multiplicidad de condiciones. La que considerábamos una ecuación de uso limitado se vuelve una herramienta conceptualmente potente. Incluso en condiciones hemodinámicas fuente de discordia entre los fisiólogos como el ejercicio, la adición de un término adicional que represente el trabajo externo sobre el sistema permite continuar utilizándola[6]. La energía total del fluido puede cambiar por las pérdidas viscosas, el trabajo externo o la transferencia de calor.

En definitiva, la ecuación combinada permite evitar la dicotomía usualmente presentada entre cada una individualmente. Adicionalmente, refuerza la importancia de los gradientes energéticos en la circulación.

\section{Otto Frank y el modelo Windkessel}

Otto Frank (1865-1944), de origen alemán, fue uno de los fisiólogos más innovadores de su época. Luego de estudiar medicina, amplió sus conocimientos en ciencias estudiando química, matemática, física y zoología. Esto le permitió realizar sus estudios y cálculos en modelos animales. Además, de la conocida ley de Frank-Starling en fisiología cardiovascular, describió la onda de pulso, su reflexión en la aorta y la relación entre la velocidad de la onda de pulso y la rigidez arterial[7]. Su trabajo precedió por muchos años y es el fundamento de tecnologías basadas en el análisis de la onda de pulso utilizadas actualmente. También es el responsable original de la descripción del modelo Windkessel.

En su modelo de dos elementos el Windkessel caracteriza y agrupa la totalidad del sistema arterial mediante dos elementos, uno de resistencia y otro de complacencia vascular[8]. Posteriormente, otros autores han propuesto modelos de tres y cuatro elementos.

El volumen sistólico es eyectado hacia la aorta que parcialmente almacena parte del mismo. Al ser un vaso distensible, actúa primero como sumidero y luego como fuente de volumen. La energía responsable del proceso sigue siendo la contracción ventricular, con una interconversión de energía cinética a potencial y viceversa garantizando el flujo diastólico. Si la aorta fuese un tubo rígido el flujo anterógrado sería únicamente sistólico con válvula aórtica abierta, y la presión de perfusión caería rápidamente a cero.

En su concepción más simple, el modelo Windkessel refuerza la noción de sectores vasculares distensibles, los dos tiempos del ciclo cardíaco y la interconversión energética.

\section{Aplicaciones clínicas}

\section{Curvas de autorregulación}

Siendo conceptualmente de suma importancia en el mantenimiento de los pacientes bajo anestesia, las curvas de autorre- 
gulación de aquellos órganos que poseen la capacidad de mantener su propio flujo presentan más sutilezas de las imaginadas en primera instancia. Tomaremos las curvas de autorregulación cerebral como objeto de análisis y ejemplo, si bien podemos sospechar distintas curvas para distintos órganos. Una situación hemodinámica suficiente para proteger el cerebro podría no serlo para el riñón.

Como se ha dicho en secciones previas, el árbol vascular está compuesto de vasos distensibles y no rígidos. Esto los hace susceptibles a ser deformados por las fuerzas físicas que actúan sobre ellos. Desde la luz de un vaso, la presión sobre su pared tenderá a aumentar su diámetro. Por lo tanto, que un órgano tenga autorregulación implica independencia del radio (y la resistencia) con respecto a la presión, al menos dentro de ciertos límites. Se constituyen así un límite superior e inferior de acuerdo a los extremos de la respuesta miogénica vasoconstrictora y vasodilatadora respectivamente. El segmento comprendido entre ambas representa la meseta de autorregulación correspondiente a un flujo determinado.

Por debajo del límite inferior de autorregulación se presenta el riesgo de hipoperfusión, isquemia e hipoxia cerebral. Este límite es aquella presión media mínima necesaria para no generar daño cerebral. Sobrepasar el límite superior tampoco se encuentra libre de riesgos, ya que implica la posibilidad de hiperperfusión y edema cerebral.

Habitualmente se presupone que el mantenimiento de los valores de presión arterial permitiendo cierto porcentaje de disminución con respecto a los basales del paciente en el preoperatorio son suficientes para tal fin. Una concepción clásica recientemente cuestionada es que la meseta de autorregulación de pacientes hipertensos se encuentra corrida a la derecha. Un estudio realizado en pacientes sometidos a bypass cardiopulmonar (y por lo tanto flujo continuo y no pulsátil) no demostró diferencias en el límite inferior de pacientes con antecedentes de hipertensión, diabetes o stroke. Distintos puntos de corte de reducción de presión arterial media no presentaron valores adecuados de sensibilidad y especificidad simultáneamente[9].

Los valores a veces citados de 60 y 150 mmHg como extremos de la curva de autorregulación normal poco tienen de "normal" en el sentido estadístico de distribución de valores en la población, ya que obvian la variabilidad interindividual entre un paciente y otro. Debemos sumar también la posibilidad de variaciones intraindividuales de acuerdo a distintos procesos fisiopatológicos. La hipocapnia y la hipercapnia resultantes de distintos seteos ventilatorios podrían modificar tanto el límite inferior, el superior y la meseta de autorregulación. Cambios en el gasto cardíaco usuales en el intraoperatorio potencialmente pueden cambiar la posición de la meseta respetando los límites de la curva, por lo que incluso manteniendo valores de presión arterial media originalmente adecuados, sigue latente el riesgo de isquemia. Distintas combinaciones dan lugar a distintas curvas, incluso en un mismo paciente. Recomendamos dos revisiones de Meng et al. acerca del tópico, con un posible modelo conceptual presentado por los autores[10],[11].

Parecería ser que, en vez de guiarse por límites arbitrarios de presión arterial media, el camino lógico es la individualización del objetivo de acuerdo al individuo y al momento quirúrgico correspondiente. Los distintos índices de autorregulación desarrollados basados en distintas técnicas, con el doppler transcraneano y el NIRS asomándose como las de mayor posibilidad de aplicación en quirófano, podrían ayudar al anestesiólogo en la personalización de la anestesia[12],[13].

\section{Hemodinamia de la población anciana}

La hemodinamia de la población envejecida presenta características propias que requieren un análisis más complejo para su descripción. Se pueden observar algunas similitudes (aunque también diferencias) con pacientes nefrópatas, ya que se está haciendo alusión a la vejez desde el punto de vista biológico y no simplemente a la edad del individuo.

La llamada insuficiencia cardíaca con fracción de eyección conservada, otrora insuficiencia diastólica, es más frecuente que la de tipo sistólica con alteraciones de la contractilidad ventricular. Lo más usual es encontrar patrones alterados tempranos de relajación prolongada en el ecocardiograma. Otro hallazgo habitual en esta población es una presión de pulso aumentada.

Como parte del envejecimiento normal se produce una disminución de la cantidad de elastina con respecto a las fibras de colágeno en la capa muscular media de los vasos, alterando sus características viscoelásticas[14]. Este proceso se denomina arterioesclerosis, término que no debe ser confundido con el de ateroesclerosis. La ateroesclerosis es, en cambio, una afectación del endotelio vascular. Si bien ambos en general coexisten, no son sinónimos.

La alteración estructural conlleva una alteración funcional, ya que la velocidad de la onda de pulso se ve aumentada. La reflexión de dicha onda (siendo estrictos, de múltiples ondas fundamentales que la conforman) es un fenómeno normal que explica, por ejemplo, las diferentes morfologías de ondas de presión invasiva radial o femoral. A mayor velocidad de onda, su rebote será más temprano en el ciclo cardíaco a nivel aórtico. En consecuencia, se produce una aumentación de la presión sistólica y de la poscarga ventricular, con una esperable hipertrofia muscular ventricular y alteraciones de los patrones de llenado diastólico. Nótese como el concepto de resistencia no es lo suficientemente abarcativo para explicar el trasfondo hemodinámico de un proceso tan frecuente como el del envejecimiento. En la génesis de la hipertensión no se encuentra un aumento del tono vascular, si no una previa alteración de índices de velocidad y reflejo de onda de pulso[15].

La aorta sufre los mismos procesos degenerativos ya descriptos, disminuyendo su distensibilidad. La función Windkessel aórtica se ve alterada, generando mayores aumentos de presión a volúmenes almacenados más pequeños, junto a un desagote aórtico más rápido. Esto contribuye a una presión de pulso elevada, junto a los fenómenos de aumentación.

Otro factor a tener en cuenta en esta población es la presencia de cierto grado de estenosis aórtica, ya que la causa degenerativa es la etiología más frecuente de esta patología.

Por todas estas razones, el paciente añoso presenta menos margen fisiológico que el joven. En el modelo del Elzinga y Westerhoff[16], el corazón se comportará un poco más como bomba generadora de presión con respecto al flujo de acuerdo a la carga impuesta. Una menor presión diastólica implica mayor riesgo de isquemia miocárdica en un corazón hipertrofiado. Las taquicardias y bradicardias tendrán mayor repercusión hemodinámica. Las primeras, por disminución del tiempo de llenado y perfusión coronaria; las segundas, por ventrículos con grados 
distintos de rigidez que ponen un límite al beneficio del aumento del tiempo diastólico y a una aorta con poca capacidad de almacenar volumen. De ser posible el anestesiólogo debe distinguir entre el paciente con relajación ventricular prolongada y aquel con un patrón restrictivo, ya que su manejo diferirá. Mientras el primero tolerará bien aumentos de la precarga requiriendo mayores tiempos diastólicos, en el segundo estará latente el riesgo de edema agudo de pulmón por sobrecarga[17].

Aún ante la rareza de un paciente anciano sano sin comorbilidades, es menester entender que las variaciones hemodinámicas serán peor toleradas y actuar en consecuencia.

\section{Injuria renal}

La injuria renal aguda es una causa importante de morbilidad hospitalaria, en especial en el período perioperatorio. Luego de la sepsis, la cirugía mayor es una de las etiologías más frecuentes en las unidades de cuidados críticos[18]. A pesar de su carga sobre el sistema de salud, se sigue buscando la mejor estrategia para prevenirla.

Una de las dificultades radica en la ausencia de herramientas diagnósticas que detecten la injuria lo más precozmente posible. Los criterios RIFLE, AKIN o KIDGO fueron un primer avance en su definición, basándose en la creatinina sérica y la oliguria dentro de cierto límite temporal. La creatinina sérica tiene baja sensibilidad, y la disminución de la diuresis baja especificidad. Se han propuesto distintos biomarcadores para superar estas limitantes, pero todavía no hay consenso en su uso[19].

La oliguria intraoperatoria no necesariamente implica una disminución del filtrado glomerular. La liberación de ADH puede ser generada por distintos estímulos extrarrenales como la nocicepción o el tipo de cirugía en sí. Por sí misma, posee un bajo valor predictivo positivo para la injuria renal aguda[20].

Como práctica actual, las guías recomiendan discontinuar agentes nefrotóxicos de ser posible, asegurar un volumen intravascular y presión de perfusión adecuada, la consideración del monitoreo hemodinámico funcional, el monitoreo de la creatinina sérica y diuresis, evitar la hiperglucemia y considerar alternativas a procedimientos con contraste[21].

Al momento, parece ser que la solución al problema seguirá siendo esquiva. Hay varias cuestiones a considerar el porqué. En primer lugar, no habría de sorprender en absoluto que las curvas de autorregulación renal se comporten de manera similar a la cerebral como se expuso previamente, aunque con distintos valores límites entre uno y otro lecho. A diferencia de los distintos índices de autorregulación cerebral elaborados, se encuentra una notoria ausencia en la literatura de algo similar en su contraparte renal, probablemente debido a la dificultad de realizar mediciones de la misma índole. La circulación renal se encuentra con el problema adicional del uso de fármacos utilizados en el perioperatorio como los IECAVARA II y los AINES que también modifican la hemodinamia renal[22].

Considerando todo esto, es poco probable que un valor único de presión arterial media logre una adecuada perfusión renal en todos los pacientes por igual, e incluso en un mismo paciente en distintos momentos del perioperatorio. Tampoco que este valor se corresponda siempre con determinado flujo sanguíneo renal. Debe considerarse también el posible efecto del aumento de las presiones venosas e intraabdominales sobre el mismo. Aun si se lograran parámetros hemodinámicos ideales para la protección renal, el uso de drogas nefrotóxicas, la injuria tisular y otros factores conspiran en contra de la misma.

\section{Discusión}

No son pocas las dificultades enfrentadas al querer intentar una explicación puramente fisiológica clásica a los dilemas hemodinámicos planteados en quirófano.

Una de ellas radica en el desconocimiento de las limitantes de distintos modelos utilizados. Otra, en que modelos más "reales" suelen ser simultáneamente más complejos. Se genera así una dicotomía entre una comprensión simple pero inacabada y otra más completa pero que muchas veces nos supera a los médicos, ya que son necesarios ciertos conocimientos del terreno de la física y la matemática. ¿Hasta qué punto adentrarnos en estos tópicos? Una sana postura sería hacerlo hasta donde sea necesario para al menos tener una noción de aquello de observación frecuente que no podemos explicar con otros paradigmas. En esta línea de pensamiento y a modo de ejemplo, las modificaciones cardiovasculares de la población geriátrica requieren al menos cierto nivel de complejidad para su descripción.

Los pacientes se comportan como una caja negra, en el sentido en que los múltiples procesos fisiológicos subyacentes se nos presentan con números de variables que suelen ser poco descriptivas. Mediciones adicionales a las de únicamente la presión arterial pueden ayudar a mejorar nuestra comprensión de los mismos, al menos desde un punto de vista exclusivamente macrohemodinámico. Es notoria la tendencia de los procesos mentales a saltar a conclusiones cognitivamente placenteras pero muchas veces erróneas en base a unos pocos datos observados, haciendo que incluso el juicio del experto pueda ser poco fiable[23]. Lamentablemente, incluso teniendo a mano múltiples monitores, los fenómenos microcirculatorios seguirán ocurriendo detrás del telón. ¿Es necesario el mismo nivel de seguimiento en todos los casos? Seguramente no, pero en el entrenamiento y la enseñanza de la Anestesiología es una sana costumbre inicialmente conocer estas variables subyacentes para después poder utilizarlas cuando sea necesario.

No todo es tan simple como la fisiología que se nos presenta en los libros. Si bien es una disciplina importante en nuestra formación, no podemos justificar absolutamente todo en lo que hacemos en base a lo que sabemos (o creemos saber) de la misma. Así nos encontramos, por ejemplo, con la posibilidad de que las curvas de autorregulación sean dinámicas y no estáticas en quirófano. Por lo tanto, son necesarios estudios fisiológicamente sensatos pero respaldados por resultados para manejar nuestra práctica. Lamentablemente, estos estudios no suelen ser la norma.

\section{Conclusiones}

Conocer la fisiología cardiovascular nunca dejará de ser relevante. Debemos tener en cuenta sus fortalezas, así como también sus limitantes. Sin importar la subespecialidad a la que se dedique, todo anestesiólogo se verá diariamente encargado de manejar la hemodinamia de los pacientes a su cargo. Una 
sólida base fisiológica ayudará a mejorar los desenlaces perioperatorios.

\section{Referencias}

1. Olivera ARE. History of the Bernoulli principle. En: Uhl T, Advances in Mechanism and Machine Science. Cham: Springer, 2019, pp.1116-78. https://doi.org/10.1007/978-3-030-20131-9_115

2. Badeer, H. Hemodynamics for medical students. Advances in physiology education 2002; 25:1-4. https://doi.org/10.1152/advances.2001.25.1.44

3. Boulpaep E. Organization of the cardiovascular system. En Boron W, Boulpaep E (ed) Medical Physiology, 3rd edition, Philadelphia: Elsevier, 2017, pp 416-420.

4. Sutera SP, Skalak R. The history of Poiseuille's law. Annual Review of Fluid Mechanics 1993; 25:1-20. https://doi.org/10.1146/annurev.fl.25.010193.000245

5. Nichols W, O'Rourke M, Vlachopoulos C. Vascular impedance. En Mc Donald's Blood Flow in Arteries. 6th edition, London: Hodder Arnold, 2011 pp 273-275. https://doi.org/10.1201/b13568

6. Synolakis $\mathrm{C}$, Badeer $\mathrm{H}$. On combining the Bernoulli and Poiseuille equation-A plea to authors of college physics texts. American Journal of Physics 1989; 57(11): 1013-19 https://doi. org/10.1119/1.15812

7. Middeke M. The pioneer in hemodynamics and pulse-wave analysis, Otto Frank. Journal of the American Society of Hypertension 2016;10(4):290-96. https://doi.org/10.1016/j.jash.2016.01.022

8. Westerhof N, Lankhaar J, Westerhof BE. The arterial Windkessel. Medical \& Biological Engineering \& Computing 2008;7(2):13141. https://doi.org/10.1007/s11517-008-0359-2

9. Joshi B, Ono M, Brown C y cols. Predicting the limits of cerebral autoregulation during cardiopulmonary bypass. Anesthesia Analgesia 2012; 114(3):503-10. https://doi.org/10.1213/ ANE.0b013e31823d292a

10. Meng L, Gelb AW. Regulation of cerebral autoregulation by carbon dioxide. Anesthesiology 2015; 122(1):196-205. https:// doi.org/10.1097/ALN.0000000000000506

11. Meng L, Hou W, Chui J. Cardiac output and cerebral blood flow: the integrated regulation of brain perfusion in adult humans. Anesthesiology 2015; 123(5):1198-208. https://doi.org/10.1097/

\section{ALN.0000000000000872}

12. Brady KM, Hudson A, Hood R y cols. Personalizing the definition of hypotension to protect the brain. Anesthesiology 2020;132(1):170-179. https://doi.org/10.1097/ ALN.0000000000003005

13. Donelly J, Budohoski K, Smielewski P y cols. Regulation of the cerebral circulation: bedside assessment and clinical implications. Critical Care 2016;20(1):129. https://doi.org/10.1186/s13054016-1293-6

14. Salvi, P. Pulse waves. How vascular hemodynamics affects blood pressure. E-book: Springer, 2012. https://doi.org/10.1007/97888-470-2439-7_3

15. Kaess B, Rong J, Larson MG y cols. Aortic stiffness, blood pressure progression, and incident hypertension. JAMA 2012; 308(9):875-881 https://doi.org/10.1001/2012.jama.10503

16. Elzinga $\mathrm{G}$, Westerhof $\mathrm{N}$. The effect of an increase in inotropic state and end diastolic volume on the pumping ability of the feline left heart. Circ Res 1978; 42:620-28. https://doi.org/10.1161/01. RES.42.5.620

17. Sharkey A, Mahmood F, Matyal R. Diastolic dysfunction - What an anesthesiologist needs to know? Best Practice \& Research Clinical Anaesthesiology 2019; 33(2):221-228. https://doi. org/10.1016/j.bpa.2019.07.014

18. Zarbock A, Koyner JL, Hoste EA y cols. Update on perioperative acute kidney injury. Anesthesia \& Analgesia 2018;127(5):12361245. https://doi.org/10.1213/ANE.0000000000003741

19. Gomelsky A, Abreo K, Khater N y cols. Perioperative acute kidney injury: stratification and risk reduction strategies. Best Practice \& Research Clinical Anaesthesiology 2020;34(2):167-182. https:// doi.org/10.1016/j.bpa.2020.04.003

20. Du Toit L, Biccard BM. The relationship between intraoperative oliguria and acute kidney injury. British Journal of Anaesthesia 2019; 6:707-710. https://doi.org/10.1016/j.bja.2019.03.008

21. KDIGO AKI Work Group. KDIGO clinical practice guideline for acute kidney injury. Kidney International Supplements 2012; 2:1138.

22. Gumbert SD, Kork F, Jackson ML y cols. Perioperative acute kidney injury. Anesthesiology 2020; 132:180-204. https://doi. org/10.1097/ALN.0000000000002968

23. Kahneman, Daniel. Pensar rápido, pensar despacio. 5ta ed., Buenos Aires: Debate, 2016. https://doi.org/10.29057/icea.v5i9.2130 